\title{
EVIDENCE FOR DIFFERENTIAL LOCALIZATION OF NORADRENALINE AND NEUROPEPTIDE Y IN NEURONAL STORAGE VESICLES ISOLATED FROM RAT VAS DEFERENS ${ }^{1}$
}

\author{
GABRIEL FRIED, ${ }^{* 2}$ LARS TERENIUS, $\ddagger$ TOMAS HÖKFELT,§ and MENEK GOLDSTEINף \\ Departments of Physiology* and Histology,§ Karolinska Institutet, S-104 01 Stockholm; Department of Pharmacology, $\ddagger$ \\ University of Uppsala, Uppsala, Sweden; and Department of Psychiatry, 1 New York University Medical Center, \\ New York, New York 10016
}

Received April 11, 1984; Revised July 9, 1984; Accepted July 23, 1984

\begin{abstract}
Using the technique of homogenization and subsequent density gradient centrifugation combined with ultrastructural analysis, the subcellular localization of noradrenaline and neuropeptide Y (NPY) was studied in vas deferens of castrated male rats. Noradrenaline showed two peaks in the gradient: one major peak at low density and another at high density. Only one NPY peak was seen, which coincided with the high density peak of noradrenaline. Electron microscopic analysis revealed high proportions of small and large vesicles in the light and heavy fractions, respectively. The present results indicate a differential subcellular localization of noradrenaline and NPY in the noradrenergic nerve endings of vas deferens. Thus, small vesicles seem to contain only noradrenaline, whereas the large vesicles may contain both noradrenaline and NPY.
\end{abstract}

Evidence has recently been obtained that neurons in both the PNS and CNS contain, in addition to its classical transmitter, a small biologically active peptide (Hökfelt et al., 1980). For some of these peptides a transmitter role has been suggested (Otsuka and Takahashi, 1977; Snyder, 1980). Thus, it is possible that neurons may release more than one messenger at their synapses. In the PNS classical transmitter and peptide in some instances may cooperate to cause a physiological response (see Lundberg and Hökfelt, 1983). In this model the classical transmitter causes a rapid response of short duration, whereas the peptide seems to evoke a long-lasting response with a slow onset. It has been speculated that there is a differential, frequency-dependent release of the two compounds (see Lundberg and Hökfelt, 1983). For the latter hypothesis it would be important to know whether or not transmitter and peptide are stored at the same place, since separate storage sites would provide a morphological basis for differential release.

Immunohistochemical studies have shown that a population of peripheral sympathetic nerves contain noradrenaline (NA) and neuropeptide Y (NPY) (Lundberg et al., 1982b). NPY is a recently isolated polypeptide with a $\mathrm{C}$-terminal amide, structurally and biologically similar to a number of peptides belong-

\footnotetext{
${ }^{1}$ This work was supported by Swedish Medical Research Council Grants 14X-07164, 04X-2887, 19X-0534, and 13X-1010; Magnus Bergvalls Stiftelse; Lars Hiertas Minne; Knut och Alice Wallenbergs Stiftelse; The Swedish Society for Medical Sciences, Karolinska Institutets Forskningsfonder; National Institute for Neurological and Communicative Disorders and Stroke Grant 06801; and National Institute of Mental Health Grant 02714. For expert technical and secretarial assistance we thank Mrs. Ingrid Dahlin, Mrs. Waldtraut Hiort, and Mrs. Elisabet Björklund.

${ }^{2}$ To whom correspondence should be addressed.
}

ing to the pancreatic polypeptide family (Tatemoto, 1982; Tatemoto et al., 1982).

Immunohistochemical studies have shown NPY-like immunoreactivity in peripheral neurons (Lundberg et al., 1982b, 1983, 1984; Edvinsson et al., 1983; Gu et al., 1983; Sundler et al., 1983; Terenghi et al., 1983), and some of these neurons are noradrenergic (Lundberg et al., 1982a, 1983, 1984), such as ganglion cells in the superior cervical stellate, and celiac ganglia as well as nerve terminals in, for example, vas deferens and heart auricles.

NA in peripheral nerves is stored in two types of vesicles, large (diameter, 70 to $100 \mathrm{~nm}$ ) and small (diameter about 50 nm) dense-core vesicles (Pellegrino de Iraldi and DeRobertis, 1961; Grillo and Palay, 1962; Richardson, 1962, 1964; Taxi, 1965; Bloom and Barrnett, 1966; see also von Euler and Hillarp, 1956). Small vesicles make up about $95 \%$ and large vesicles about $5 \%$ of the vesicles in sympathetic terminals on small rodents such as rats and guinea pigs (Hökfelt, 1969; Fillenz, 1971; Fried, 1981; Thureson-Klein, 1983). The intraneuronal storage site for NPY so far is unknown. Therefore, we have analyzed the subcellular distribution of NPY in relation to NA in rat vas deferens, an organ with dense sympathetic innervation (Sjöstrand, 1965; Norberg et al., 1966) that has previously been used as a source for isolation of noradrenergic vesicles (Bisby and Fillenz, 1971; Fried et al., 1978; Fried, 1981).

\section{Materials and Methods}

Immunohistochemistry. Normal male and castrated ( 6 to 8 weeks) male rats (body weight, 200-250 gm; pathogen-free strain; Anticimex, Stockholm, Sweden) were anesthetized with mebumal $(40 \mathrm{mg} / \mathrm{kg}$, i.p.) and perfused via the ascending aorta with $100 \mathrm{ml}$ of $0.9 \%$ sodium chloride followed by ice-cold $10 \%$ formalin (Pease, 1962) for $30 \mathrm{~min}$. The vasa deferentia were dissected out, immersed in the same fixative for $90 \mathrm{~min}$, rinsed in $0.1 \mathrm{M}$ phosphate buffer with $10 \%$ sucrose added 
for at least $24 \mathrm{hr}$ and processed for indirect immunofluorescence according to the method of Coons and collaborators (see Coons, 1958). Briefly, adjacent sections were incubated for 18 to $24 \mathrm{hr}$ at $4^{\circ} \mathrm{C}$ with rabbit antisera to NPY (102 B; see Lundberg et al., 1984) diluted 1:400 or to tyrosine hydroxylase (TH) (see Markey et al., 1980) diluted 1:400, rinsed in phosphate-buffered saline (PBS), incubated in fluorescein isothiocyanate-conjugated swine anti-rabbit antibodies (Dakopatts, $\mathrm{Co}-$ penhagen, Denmark) diluted 1:10, rinsed in PBS, mounted in glycerine:PBS (3:1), and examined in a Zeiss Standard fluorescence microscope equipped with proper filter combinations.

Electron microscopy of intact tissue. Rats of the same groups as described above were anesthetized with mebumal $(40 \mathrm{mg} / \mathrm{kg}$, i.p.) and small pieces of vas deferens were immersed in $3 \%$ potassium permanganate (Richardson, 1966) for $2 \mathrm{hr}$, rinsed in Ringer's solution, contrasted en bloc with 2\% uranyl acetate in Ringer's solution for 4 to 6 $\mathrm{hr}$, and embedded and examined as described below. For further details on the technique, see Hökfelt (1968).

Rats (as above) were perfused under mebumal anesthesia (as above) via the ascending aorta with $50 \mathrm{ml}$ of Tyrode's buffer followed by $1 \%$ glutaraldehyde (Merck, Darmstadt) in $0.1 \mathrm{M}$ phosphate buffer ( $\mathrm{pH} 7.2$ to 7.4) for $30 \mathrm{~min}$. Small pieces of vas deferens were taken out and immersed in the same fixative for 30 to $90 \mathrm{~min}$, followed by immersion in $0.2 \mathrm{M}$ sodium chromate buffer $(\mathrm{pH} 6.0$ ) for 2 to $18 \mathrm{hr}$ (Tranzer and Richards, 1976), postfixed in $2 \%$ osmium tetroxide, dehydrated, embedded, and examined as described below.

Subcellular fractionation. Vasa deferentia $(0.7$ to $1.0 \mathrm{gm})$ from about 20 rats (body weight, $200 \mathrm{gm}$; pathogen-free strain; Anticimex), castrated 6 to 8 weeks before killing, were used in each experiment. Organs were homogenized in $10 \mathrm{vol}$ of ice-cold $0.25 \mathrm{M}$ sucrose with $10 \mathrm{mM}$ potassium phosphate, $\mathrm{pH}$ 7.3. The homogenate was subjected to differential and density gradient centrifugation using procedures similar to those described previously (Fried, 1981).

Briefly, the homogenate was centrifuged at $800 \times g$ for $10 \mathrm{~min}$ in a Sorvall refrigerated centrifuge. The pellet (P1) containing nuclei and cell debris was removed, and the supernatant (S1) was centrifuged at $20,000 \times g$ for $30 \mathrm{~min}$. The second pellet (P2), containing most of the mitochondria, was removed and the second supernatant (S2) was put on a linear density gradient $(0.25$ to $1.2 \mathrm{M})$ and centrifuged at 280,000 $\times g_{\max }$ for 90 min in a Beckman L62B ultracentrifuge using an SW40 rotor. After centrifugation the gradient was divided into 7 to 12 equal fractions, which were removed from above using hooked Pasteur pipettes (Fig. 1). For biochemical analysis, fractions were diluted to isotonicity and centrifuged at $140,000 \times g_{\max }$ for $45 \mathrm{~min}$ in order to separate sedimentable from soluble material. Pellets were resuspended in $1 / 2$ to 1 vol of ice-cold water. NA was determined by high pressure liquid chromatography with electrochemical detection (Hjemdahl et al., 1979). NPY was determined by radioimmunoassay (Lundberg et al., 1984).

The density gradient fractions as well as the starting material (S2) were analyzed in the electron microscope. The fractions (volume, 700 to $1000 \mu \mathrm{l}$ ) were diluted up to $2 \mathrm{ml}$ with ice-cold $5 \%$ glutaraldehyde in $0.1 \mathrm{M}$ phosphate buffer (pH 7.2 to 7.4) in polycarbonate tubes (Beckman Instruments, Palo Alto, CA) and centrifuged at $145,000 \times g_{\max }$ for 30 min. After fixation for 2 to $4 \mathrm{hr}$, the pellets were rinsed in $0.01 \mathrm{M}$ phosphate buffer ( $\mathrm{pH} 7.2$ ), immersed in $2 \%$ osmium tetroxide for $2 \mathrm{hr}$, rinsed in Ringer's solution, dehydrated in increasing concentrations of ethanol, cleared in propylene oxide, and embedded in Epon. Ultrathin sections were cut in a Reichert OmU3 ultratome, contrasted by uranyl acetate and lead citrate (Reynolds, 1963), and examined in a Philips 300 electron microscope. The diameters of vesicular structures were studied on micrographs taken at a magnification of $\times 57,000$ with a Zeiss TGZ3 particle size analyzer.

\section{Results}

Immunohistochemistry. After incubation of adjacent sections of vas deferens with antibodies to NPY and TH, dense overlapping networks of strongly immunoreactive fibers were observed in the muscle layers (Fig. 2, $a$ and $b$ ). In addition, fluorescent NPY- and TH-positive fibers were seen around blood vessels around vas deferens (Fig. 2, $a$ and $b$ ). Adjacent axon bundles were strongly TH and moderately strongly NPY positive (Fig. $2, a$ and $b$ ). In addition, a loose plexus of NPY-immunoreactive fibers was seen in the connective tissue under the epithelium

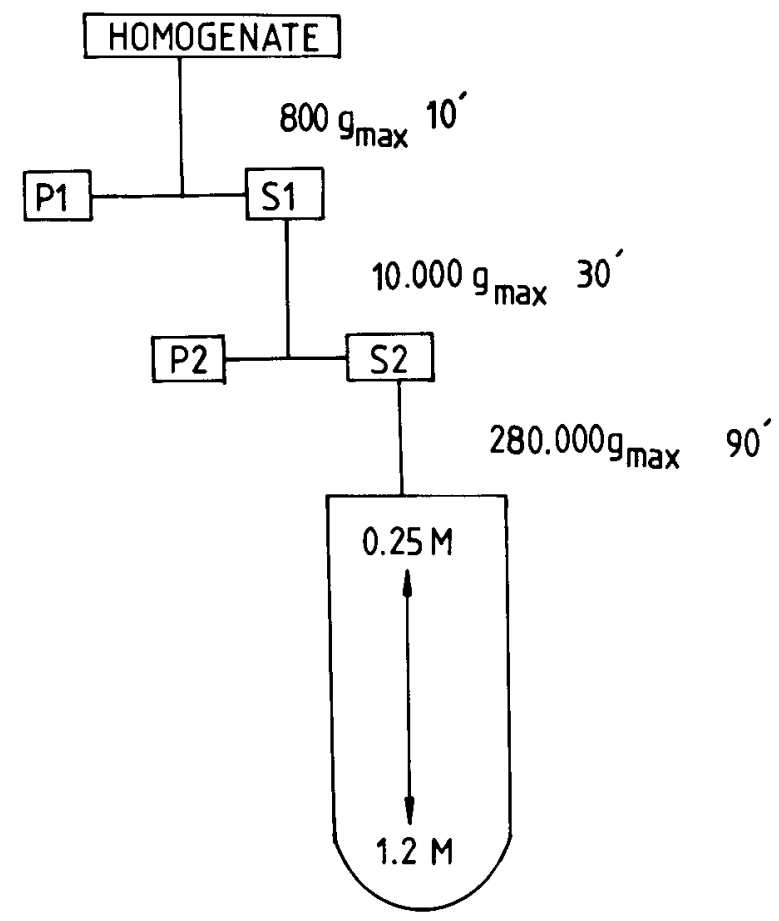

Figure 1. Fractionation procedure used in the studies on vas deferens of castrated rat. The homogenate was centrifuged at $800 \times g_{\max }$ for 10 min; the supernatant was centrifuged at $10,000 \times g_{\max }$ for $30 \mathrm{~min}$, and the supernatant of the centrifugation (S2) was layered on linear sucrose density gradients $(0.25$ to $1.2 \mathrm{M})$, which were centrifuged at $280,000 \times$ $g_{\max }$ for $90 \mathrm{~min}$. The gradient was divided into 7 to 12 equal fractions and analyzed.

(Fig. 2c). No corresponding TH-positive networks could be seen (Fig. $2 d$ ).

Electron microscopy of intact tissue. In glutaraldehyde $/ \mathrm{OsO}_{4}{ }^{-}$ fixed material, nerve endings containing small and large densecore vesicles were observed. Whereas the density and size of the cores in the large vesicles mostly were similar in all vesicles, the small ones often had a core of varying size, and some small vesicles lacked a core. In permanganate-fixed material the smallest vesicles had an electron-dense core (Fig. 3, $a$ and $b$ ). The large vesicles had a homogeneous interior of either a moderate or low electron density (Fig. $3 a$ ) but could also contain a strongly electron-dense core of varying size (Fig. $3 b$ ).

Biochemistry of subcellular fractions. The homogenate contained $20.6 \pm 7.4 \mathrm{pmol}$ of NPY/mg of progein and $1596 \pm 152$ $\mathrm{pmol}$ of $\mathrm{NA} / \mathrm{mg}$ of protein. When related to organ weight, the values were $0.29 \pm 0.16 \mathrm{nmol}$ of $\mathrm{NPY} / \mathrm{gm}$ of wet weight and $27.2 \pm 3.9 \mathrm{nmol}$ of NA/gm of wet weight $(n=3$; values $\pm \mathrm{SEM})$. The molar ratio of N $\Lambda$ to NPY was $77: 1$ in the homogenate.

After differential centrifugation, $14.1 \%$ of the NPY remained in the $\mathrm{P} 1$ pellet and $16.5 \%$ remained in the $\mathrm{P} 2$ pellet as compared to $11.6 \%$ and $7.6 \%$ of the NA. In $\mathrm{S} 2,69.4 \%$ of the NPY remained, as compared to $78.4 \%$ of the NA (Table I). Of the total amount of NPY that was put on the gradient, $61 \%$ was recovered in a sedimentable form after centrifugation at $140,000 \times g_{\max }$ for $45 \mathrm{~min}$. The corresponding figure for NA was $39 \%$.

In the density gradient, NPY showed a single peak in a high density fraction (corresponding to $0.86 \mathrm{M}$ sucrose). 'This fraction contained $11.9 \pm 3.9 \mathrm{pmol}$ of NPY $/ \mathrm{ml}$. The specific activity was $35.9 \pm 11.2 \mathrm{pmol} / \mathrm{mg}$ (Fig. 1).

NA showed two peaks in the gradient, one at lower $(0.68 \mathrm{M})$ and one at higher $(0.86 \mathrm{M})$ density. The high density peak coincided with the NPY peak (Fig. 4). The light NA peak 

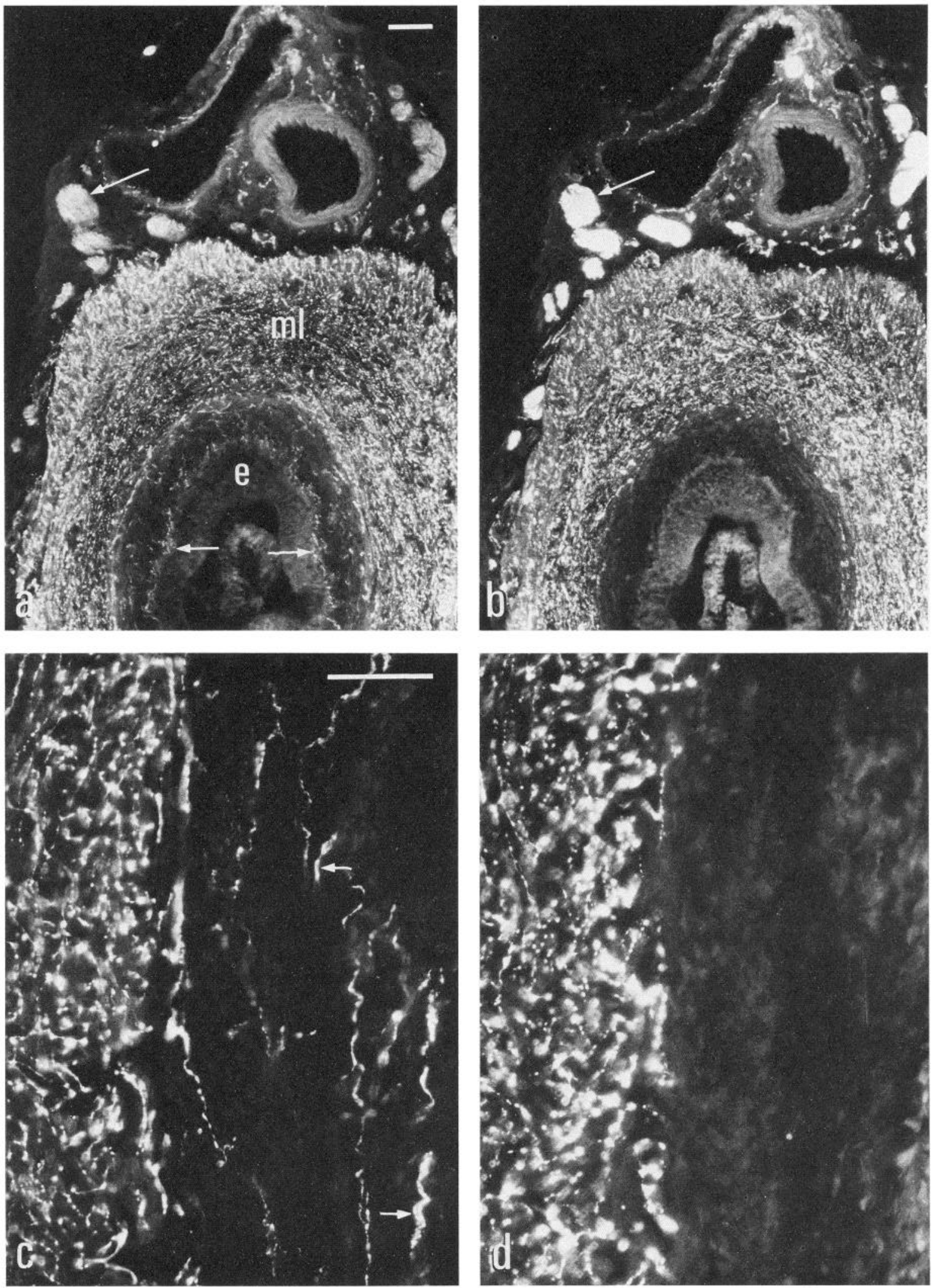

Figure 2. Immunofluorescence micrographs of the vas deferens after incubation with antiserum to NPY $(a$ and $c)$ and to TH $(b$ and $d)$. $a$ and $b$ as well as $c$ and $d$ show adjacent sections, respectively. Dense overlapping networks of NPY-and TH-immunoreactive fibers can be seen in the muscle layers $(\mathrm{ml})$. Axon bundles (long arrows) surrounding vas deferens are immunoreactive to both substances and positive fibers can also be seen around blood vessels. Note that NPY-immunoreactive fibers (short arrows in $a$ and $c$ ) can be seen just under the epithelium (e), and no corresponding TH-immunoreactive fibers are present $(b$ and $d)$. Bars indicate $50 \mu \mathrm{m}$. 
TABLE I

Differential centrifugation of rat vas deferens

The distribution of NA and NPY in fractions P1, P2, and S2 is shown, expressed as both percentage and absolute value of the total amount recovered in $\mathrm{P} 1, \mathrm{P} 2$, and $\mathrm{S} 2$. The mean of three experiments is given as values \pm SEM. Recovery for NA was $98 \%$, for NPY, $87 \%$, and for protein, $83 \%$.

\begin{tabular}{lrrrr}
\hline & P1 & P2 & S2 & P1+ P2 + S2 \\
NA & & & & \\
Percentage & $11.6 \pm 0.7$ & $7.6 \pm 1.3$ & $78.4 \pm 4.0$ & 100 \\
Picomoles & $4,522 \pm 759$ & $2,857 \pm 410$ & $30,209 \pm 4,739$ & $38,242 \pm 5,175$ \\
NPY & & & & \\
Percentage & $14.1 \pm 4.9$ & $16.5 \pm 1.6$ & $69.4 \pm 6.2$ & 100 \\
Picomoles & $23.4 \pm 7.8$ & $28.7 \pm 4.2$ & $118.7 \pm 59$ & $170.8 \pm 8.6$ \\
\hline
\end{tabular}

contained $2100 \pm 412 \mathrm{pmol} / \mathrm{ml}$ and the heavy peak contained $500 \pm 104 \mathrm{pmol} / \mathrm{ml}$.

Corresponding values for specific activity were $22,443 \pm 3,625$ $\mathrm{pmol} / \mathrm{mg}$ and $3,095 \pm 1,747 \mathrm{pmol} / \mathrm{mg}$. The molar ratio of NA to NPY in the heavy fraction was $46: 1$.

The relative enrichment in the fractions, expressed as ratio of specific activity (picomoles per milligram) in the fractions over that of the homogenate, is given in Table II. NA is enriched about 30 -fold in fraction 5 . NPY is enriched about 2 -fold in fractions 6 to 8 . The recoveries in the gradient were $70 \%$ for NA, $65.1 \%$ for NPY, and $87 \%$ for protein.

Electron microscopy. The $\mathrm{S} 2$ pellet contained a heterogenous collection of vesicles of various sizes and of various densities as well as ribosomes, lysosome-like structures, membrane fragments, and mitochondria (Fig. 3c). Fraction $1(0.27 \mathrm{M})$ was characterized by high numbers of ribosome-like structures (Fig. $3 d$ ) and, in its upper part, small numbers of vesicles of varying size. Also in fraction 2, numerous ribosomes were still observed but with an increasing number of vesicles (Fig. $3 e$ ). Fractions 3 to 8 all contained numerous vesicles of various sizes and varying electron density as well as membrane fragments often forming rounded structures (Fig. $3, f$ to $i$ ). In fraction 8 an increasing number of club-shaped, elongated, electron-dense structures, as well as lipid droplets and mitochondria, were found (Fig. 3i). The quantitative analysis revealed that the vast majority of particles in fraction 1 had a diameter of about 150 to $200 \mathrm{~nm}$. In fractions 3 and 4 most vesicles had a diameter of about 500 to $550 \mathrm{~nm}$. Fraction 5 also contained numerous vesicles in this size range; many of them, about $50 \%$, had a

\section{TABLE II}

Relative enrichment in fractions expressed as ratio of specific activity (picomoles per milligram of protein) in fraction to that of the homogenate

For description of fractions, see "Materials and Methods" and Figure 1. The molarity (M) of the fractions is given within parentheses. In the third column, the molar ratio of NA to NPY is given. Values are mean of three experiments \pm SEM.

\begin{tabular}{lclc}
\hline Fraction $(M)$ & NA & NPY & NA:NPY $(\mathrm{mol} / \mathrm{mol})$ \\
\hline Homogenate & 1 & 1 & 77 \\
P1 & 0.5 & 0.08 & 229 \\
P2 & 0.7 & 0.3 & 92 \\
S2 & 1.9 & 0.6 & 125 \\
$1(0.27)$ & 1.2 & 0.1 & 430 \\
$2(0.34)$ & 2.6 & 0.2 & 590 \\
$3(0.49)$ & 5.0 & 0.2 & 892 \\
$4(0.61)$ & 16.4 & 0.6 & 1107 \\
$5(0.68)$ & 28.1 & 1.2 & 905 \\
$6(0.73)$ & 14 & 1.8 & 300 \\
$7(0.86)$ & 3.9 & 1.7 & 86 \\
$8(0.96)$ & 2.2 & 1.9 & 46 \\
$9(1.04)$ & 1.2 & 0.8 & 61 \\
$10(1.2)$ & 1.1 & 1.0 & 96 \\
\hline
\end{tabular}

diameter between 440 and $560 \mathrm{~nm}$ (Fig. 5), and of these vesicles about $10 \%$ had an electron-dense core. The heavier fractions had a higher proportion of larger vesicles (Fig. 5). For example, in fraction 7 most vesicles had a diameter of about 625 to 750 $\mathrm{nm}$, but also, many vesicles with a diameter ranging between 800 and $1100 \mathrm{~nm}$ were seen (Fig. 5). In fraction 7 about $20 \%$ of the vesicles had a dense core.

\section{Discussion}

The present findings confirm that the vast majority of the noradrenergic nerve endings in the vas deferens contain an NPY-like peptide (Lundberg et al., 1982b). In addition, a small population of nerves under the epithelium may contain NPYlike immunoreactivity but no catecholamines. Their number is very small when compared to the NA/NPY-immunoreactive fibers in the muscle layers. Therefore, NPY-like immunoreactivity present in these fibers in all probability do not influence the major conclusions drawn from the subcellular fractionation experiments in this study.

Many earlier electron microscopic studies on intact tissue, as well as the present one, have demonstrated the presence of two types of vesicles in peripheral noradrenergic nerve endings including vas deferens containing, respectively, small and large dense-core vesicles (Grillo and Palay, 1962; Richardson, 1962, 1964; Taxi, 1965). There is general agreement that the dense core in the small vesicles in all probability reflects the presence of NA. This is supported by depletion experiments using drugs such as reserpine which completely prevent the appearance of the small dense cores in vivo (Hökfelt, 1966; Van Orden et al., 1966, 1967; Tranzer and Thoenen, 1967; Farrell, 1968), as well as by using drugs which enhance the electron density in the vesicles (Tranzer and Thoenen, 1967, 1968). With regard to the large dense-core vesicles, the situation is more complex, and the electron microscopic appearance depends on the fixation technique. As shown in this and earlier studies, many large vesicles in $\mathrm{KMnO}_{4}$-fixed tissue contain a strongly electrondense core probably reflecting the presence of NA at the moment of fixation (Hökfelt, 1968). However, studies on glutaraldehyde $/ \mathrm{OsO}_{4}$-tixed material suggest that components other than the amine are essential for the electron density, although NA may contribute to the density of the core (Hökfelt, 1966, 1968, 1973; Bloom and Aghajanian, 1968; Pfenninger, 1973; Bloom, 1972; Thureson-Klein et al., 1974; Fried et al., 1978, 1981a, b; Fried, 1981; Pollard et al., 1982; 'Thureson-Klein, 1983).

In agreement with earlier studies (Bisby, 1971; Bisby and Fillenz, 1971; Fried et al., 1978), the present subcellular analysis in sucrose density gradients demonstrates the presence of NA in both a low and a high density fraction. In contrast, NPYlike immunoreactivity is contained only in a subcellular organelle with sedimentation characteristics similar to those of large noradrenergic vesicles. In gradients of the type used $(0.25$ to $1.2 \mathrm{M}$ sucrose), such vesicles typically equilibrate at 0.8 to 1.1 

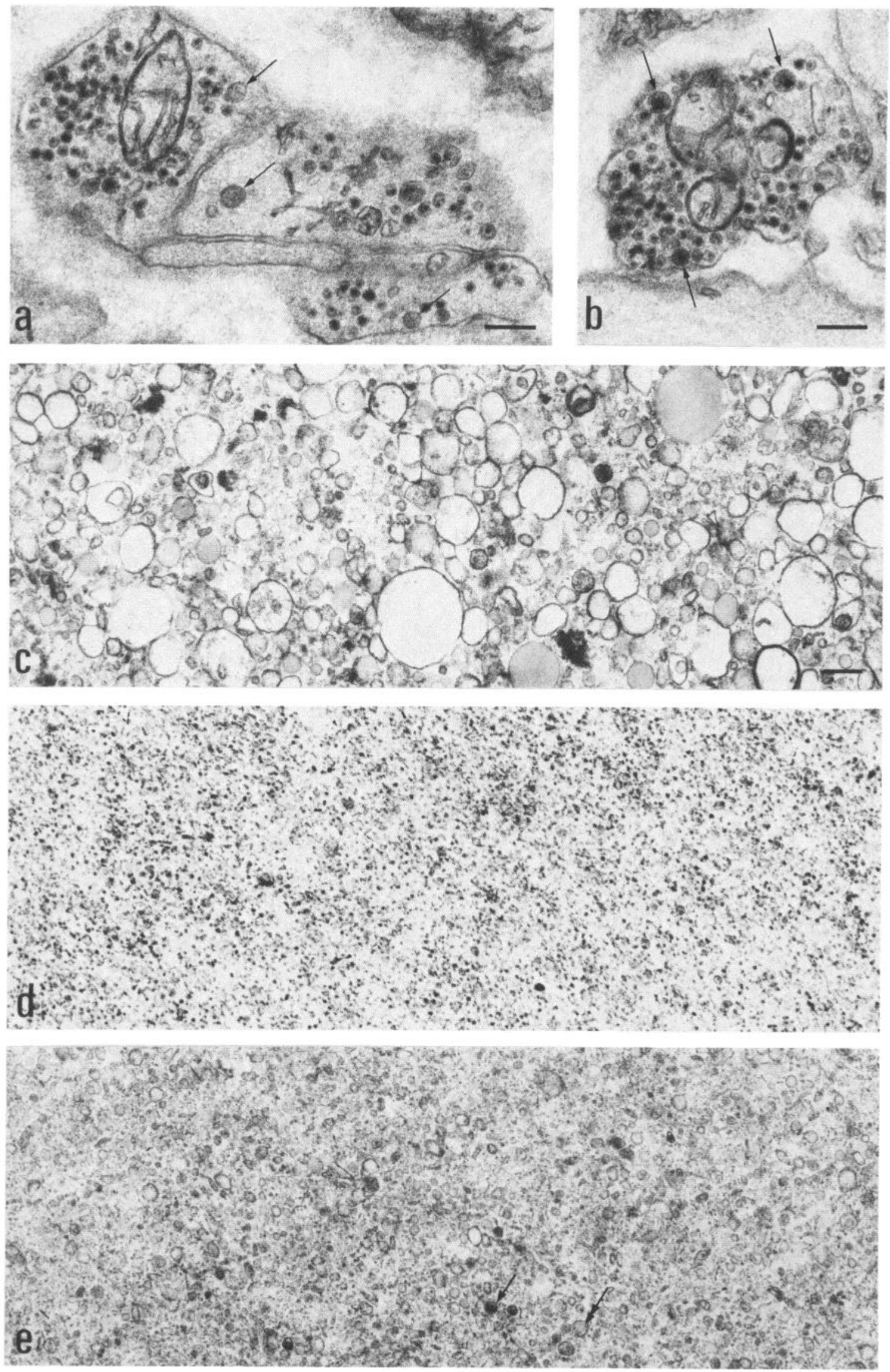

Figure 3. Electron micrographs of nerve endings in intact, potassium permanganate-fixed vas deferens $(a$ and $b)$ and of various subcellular fractions ( $c$ to $i$, fraction $\mathrm{S} 2$, fraction 1, fraction 2, fraction 3, fraction 5, fraction 7, and fraction 8, respectively). $c$ to $i$ show glutaraldehyde/ osmium tetroxide-fixed pellets. $a$ and $b$, The nerve endings contain many small dense-core vesicles and fewer large vesicles, some of which have an interior with a low electron density (arrows in $a$ ), whereas others (arrows in $b$ ) have a strongly electron-dense core of varying size. $c$ to $i$, The S2 fraction contains a heterogeneous population of structures including small vesicles, membrane fragments forming round sacs, and lipid droplets. Numerous ribosomes are seen in fraction $2(d)$, and fraction $3(e)$ contains increasing numbers of vesicles with $(a r r o w)$ and without 

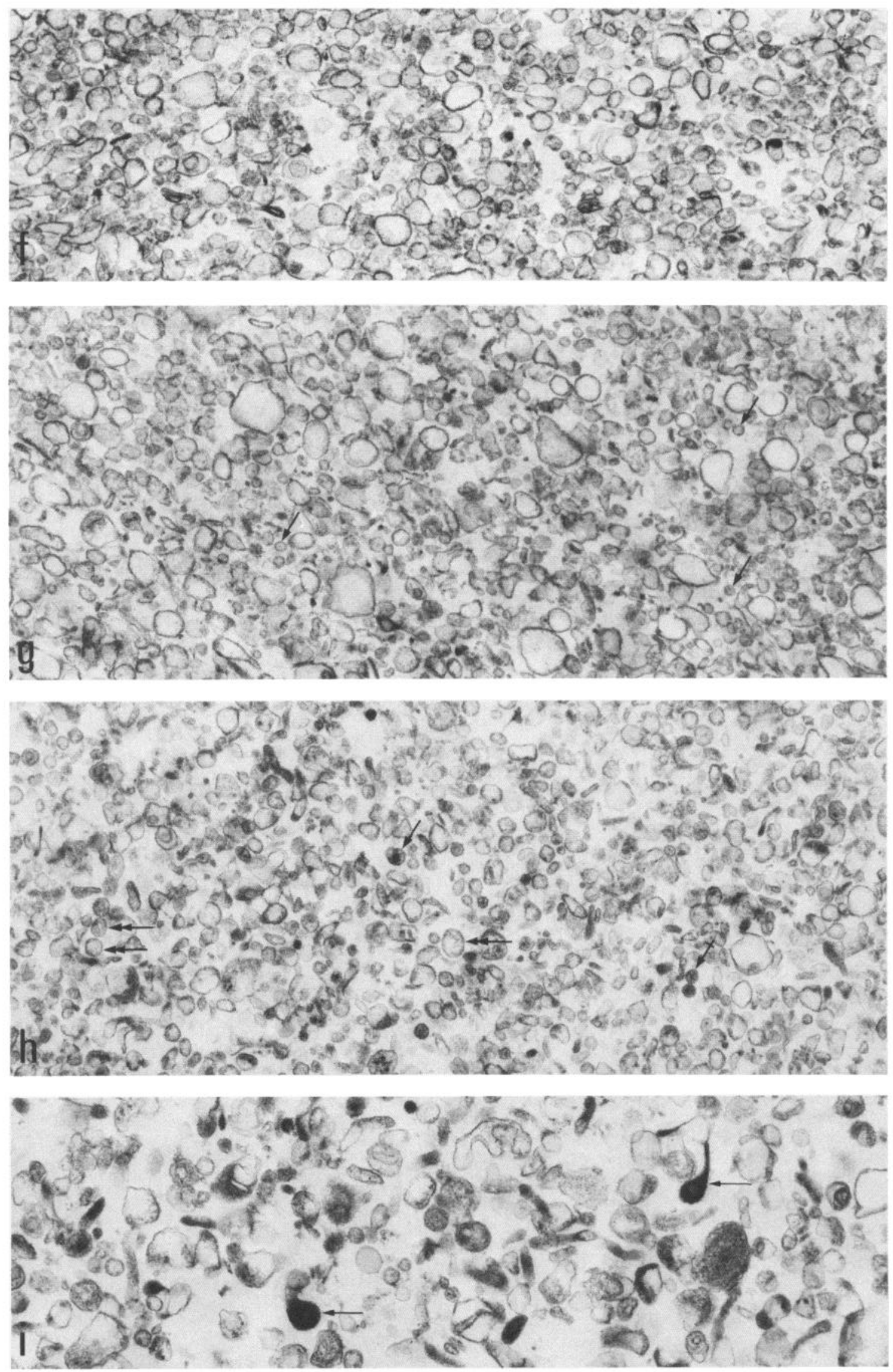

(double-headed arrow) electron-dense interior. Fractions $3(f)$ and $5(g)$ contain numerous vesicles, many of which have a diameter of about 500 $\mathrm{nm}$ (arrows in $g$ ), as well as many membranes forming rounded sac-like structures. Fraction $7(h)$ appears fairly homogeneous with many large vesicles with (arrow) or without (double-headed arrows) dense cores as well as many smaller vesicles with dense cores (crossed arrow). Fraction 8 (i) is heterogeneous with club-shaped, strongly electron-dense structures (arrows), elongated sacs, and vesicles of varying size. Bars indicate $200 \mathrm{~nm} . c$ to $i$ have the same magnification. 


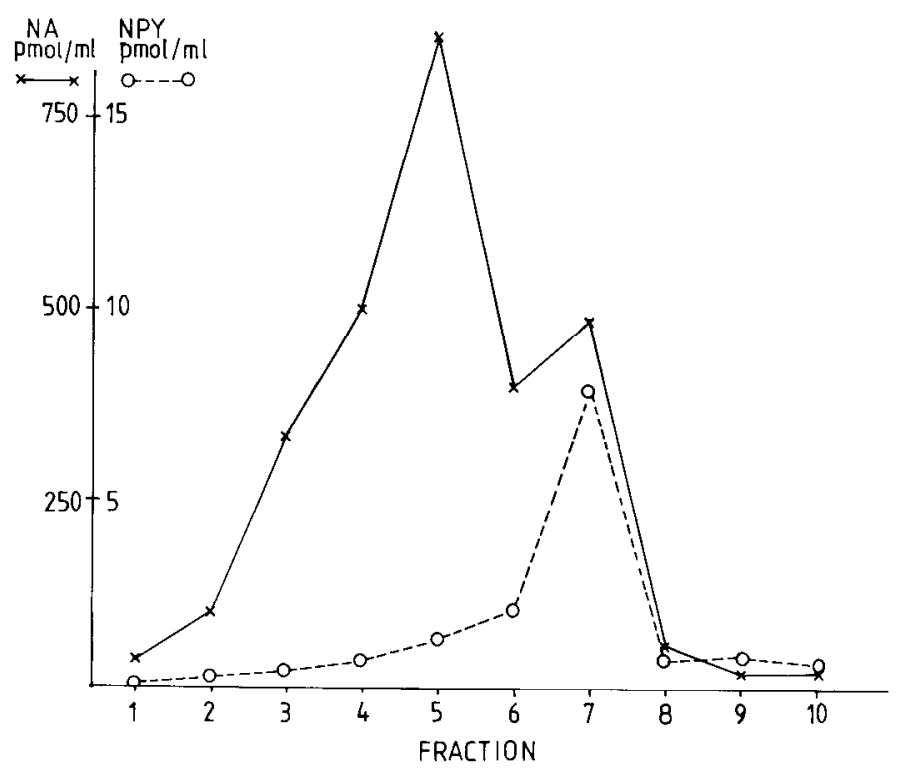

Figure 4. Subcellular distribution of NA $(x)$ and NPY $(O)$ in a density gradient of rat vas deferens. On the abscissa, totally recovered sedimentable substance is given as picomoles per milliliter after centrifugation at $145,000 \times g_{\max }$ for $45 \mathrm{~min}$. On the ordinate, density gradient fractions 1 to 10 are given, corresponding to the following sucrose molarities: $1(0.26 \mathrm{M}), 2(0.32 \mathrm{M}), 3(0.47 \mathrm{M}), 4(0.56 \mathrm{M}), 5(0.69$ M), $6(0.74 \mathrm{M}), 7(0.84 \mathrm{M}), 8(0.91 \mathrm{M}), 9(0.98 \mathrm{M}), 10(1.2 \mathrm{M})$. Recoveries of NA $=70 \%$, of $\mathrm{NPY}=65 \%$, and of protein $=87 \%$. One representative gradient out of three similar ones is shown.

M sucrose, depending on the time and $\mathrm{G}$ force of centrifugation (Bisby, 1971; Bisby and Fillenz, 1971).

The electron microscopic analysis of the different fractions revealed that the highest proportion of small vesicles was found in fraction 5 ( 0.5 to $0.65 \mathrm{M})$, whereas the highest proportion of large vesicles was observed in fraction $7(0.8$ to $0.9 \mathrm{M})$. Only a rather small number of vesicles in these fractions had an electron-dense core. This is in all probability due to the fact that the amine as well as other components of the vesicular matrix have been lost during the homogenization and centrifugation procedures. In contrast to some previous studies (Fried et al., 1978, 1981b), no attempts were made to restore the electron-dense core by incubation with NA- $\mathrm{Mg}^{2+}$-ATP.

The co-distribution of the heavy NA peak and the NPY peak may indicate that NPY is stored together with NA in large vesicles, or that they are stored in different organelles with similar size and density (Fig. 6). The latter hypothesis receives apparent support by electron micrographs of $\mathrm{KMnO}_{4}$-fixed nerve endings demonstrating some large vesicles with low and others with high electron density, as shown in this paper. However, administration of exogenous amines in vivo and in vitro enhances electron density of all large vesicles (Tranzer and Thoenen, 1967; Hökfelt, 1968), suggesting that large vesicles in noradrenergic neurons in general have NA-storing capacity. Thus, it seems most likely that NPY is stored together with NA in large vesicles.

The distribution of NPY in the differential centrifugation was somewhat different from that of NA. In the S2 supernatant, $69.4 \%$ of the NPY was still present, as compared to $78.4 \%$ of the NA. After centrifugation of $\mathrm{S} 2$ for $140,000 \times g_{\max }$ for 45 min, $61 \%$ of the NPY was sedimentable as compared to $39 \%$ of the NA. Since there probably are two storage compartments for NA (large and small vesicles), but apparently only one for NPY, the distribution would be expected to be different. A higher proportion of sedimentable NPY as compared to NA is also compatible with the hypothesis of storage of NPY only in large (heavy) vesicles.

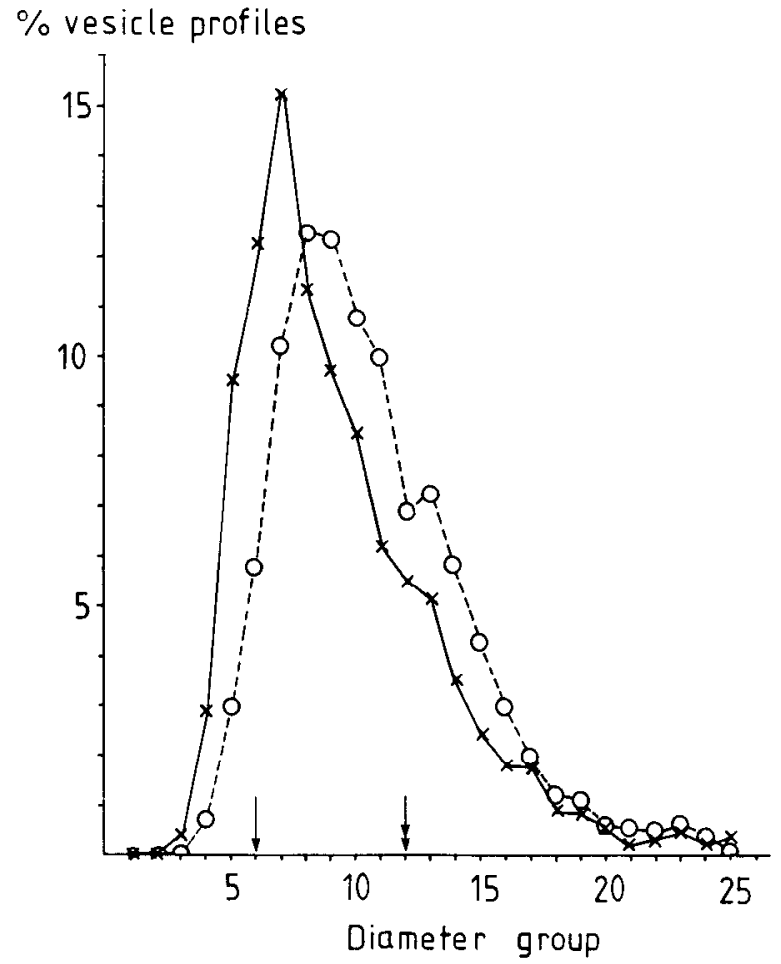

Figure 5. Distribution (percentage) of the diameters of vesicle profiles in subcellular fractions of vas deferens of castrated male rats. Fraction $5(0.69 \mathrm{M}$ sucrose) $(x)$ and fraction $7(0.84 \mathrm{M}$ sucrose $)(O)$ are shown. In fraction 5,2756 vesicular profiles were counted (mean, 644/picture), and in fraction 7,1929 vesicular profiles were counted (mean, 482) picture). Values shown are the mean of four pictures per group (SEM was less than $5 \%$ ). The diameters of the vesicular profiles have been grouped in the particle size analyzer, and the means of the groups are as follows (in nanometers): group 1, 63; group 2, 125; group 3, 250; group 4, 375; group 5, 438; group 6, 500; group 7, 563; group 8, 625; group 9,750 ; group 10, 813; group 11, 875; group 12, 1000; group 13, 1063; group 14, 1125; group 15, 1188; group 16, 1250; group 17, 1313; group 18, 1375; group 19, 1438; group 20, 1500; group 21, 1563; group 22,1625 ; group 23, 1688; group 24, 1750; group 25, 1875. The arrow indicates $500 \mathrm{~nm}$ and the double-headed arrow indicates $1000 \mathrm{~nm}$.

The ratio of NA to NPY on a molar basis was about 50:1 in fraction 8 , containing large vesicles. This is remarkably similar to the ratios of classical transmitter to neuropeptide found in other coexistence systems investigated by the technique of subcellular fractionation. In bovine splenic nerve, the ratio of NA to enkephalin in a large dense-core vesicle fraction was 60:1 (Wilson et al., 1980; Fried et al., 1981a). In cat submandibular gland, the ratio of acetylcholine to VIP was 70:1 in a fraction enriched in VIP, acetylcholine, and large dense-core vesicles (Lundberg et al., 1981).

The specific activity of NA was markedly increased in the small dense-core vesicle fraction, about 30 -fold higher than that in the homogenate. The specific activity of NPY was maximally increased in the heavy fraction $(0.86 \mathrm{M})$ but only 2 fold higher than that in the homogenate. However, the increase in specific activity of NA in this fraction was also about 2-fold, i.e., comparable to that of NPY. This indicates a similar degree of enrichment of NPY and NA stored in large vesicles. In addition, the observed 30 -fold enrichment of NA in the small vesicle fraction as compared to a 2 -fold enrichment in the large vesicle fraction fits rather well with the calculated ratio of small $(95 \%)$ to large $(5 \%)$ vesicles in the sympathetic terminals of rat vas deferens (see Thureson-Klein, 1983).

The results provide further evidence of compartmentalization of coexisting neurotransmitter/neuropeptides at the sub- 


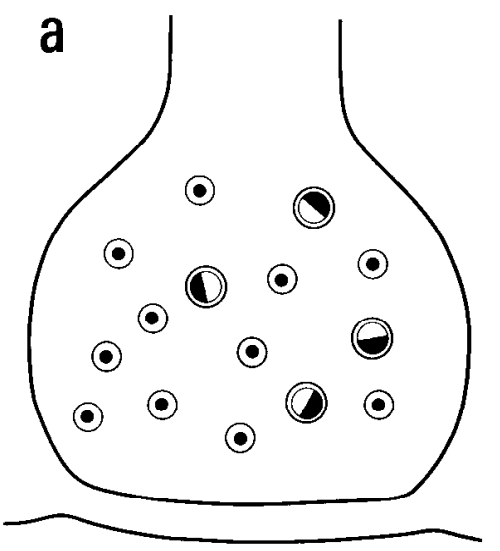

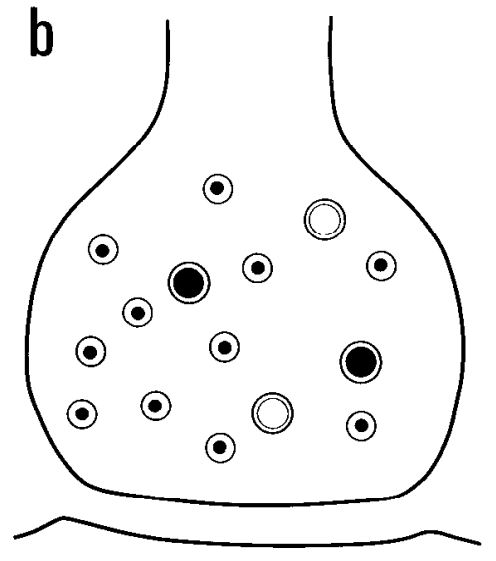

Figure 6. Schematic illustration of the storage sites of NA and NPY in a nerve ending of rat vas deferens based on the present experiments. The small vesicles store only NA, whereas the large vesicles contain both NA and NPY (a). However, a second alternative $(b)$ cannot be excluded; NA and NPY are stored in separate large vesicles.

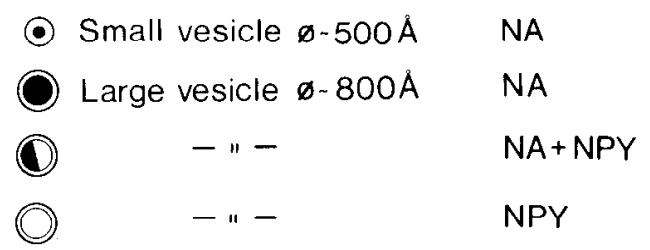

cellular level (Wilson et al., 1980; Fried et al., 1981a, 1984; Lundberg et al., 1981; Klein et al., 1982; Lundberg and Hökfelt, 1983). The occurrence of both large and small NA storage organelles has long been regarded as an enigmatic feature of adrenergic neurons. It seems possible that one important function of the large vesicles is storage of neuropeptide(s), as suggested also from recent immunoelectron microscopic studies on peripheral peptide neurons (Probert et al., 1981; Tapia et al., 1983), whereas the small vesicles primarily store the classical transmitter (Fried, 1982; Fried et al., 1984). This may provide a morphological basis for separate control mechanisms for differential release. Evidence that NA and NPY may cooperate to produce the biological response to sympathetic nerve stimulation has been obtained by in vivo experiments in the cat, where infusion of NA and NPY together, but not NA or NPY alone, mimicked the vascular effects of sympathetic nerve stimulation (Lundberg and Tatemoto, 1982).

\section{References}

Bisby, M. A. (1971) Studies on transmitters. Doctoral thesis, Oxford University, Oxford, England.

Bisby, M. A., and M. Fillenz (1971) The storage of endogenous noradrenaline in sympathetic nerve terminals. J. Physiol. (Lond.) 215: 163-179.

Bloom, F. E. (1972) Electron microscopy of catecholamine-containing structures. In Handbook of Experimental Pharmacology. Catecholamines. Vol. 33: H. Blaschko and E. Muscholl, pp. 46-78, SpringerVerlag, Heidelberg.

Bloom, F. E., and G. K. Aghajanian (1968) An electron microscopic analysis of large granular synaptic vesicles of the brain in relation to monoamine content. J. Pharmacol. Exp Ther. 159: 261-273.

Bloom, F. E., and R. J. Barrnett (1966) Fine structural localization of noradrenaline in vesicles of autonomic nerve endings. Nature 210: $599-601$.

Coons, A. H. (1958) Fluorescent antibody methods. In General Cytochemical Methods, J. F. Danielli, ed., pp. 399-422, Academic Press, Inc., New York.

Edvinsson, L., P. Emson, J. McCulloch, K. Tatemoto, and X. Uddman (1983) Neuropeptide Y: Cerebrovascular innervation and vasomotor effects in the cat. Neurosci. Lett. 43: 79-84.

Farrell, K. E. (1968) Fine structure of nerve fibers in smooth muscle of the vas deferens in normal and reserpinized rats. Nature New Biol. 217. 279-281.

Fillenz, M. (1971) Fine structure of nodradrenaline storage vesicles in nerve terminals of rat vas deferens. Philos. Trans. R. Soc. Lond. (Biol) 261: 319-323.

Fried, G. (1981) Small noradrenergic storage vesicles isolated from rat vas deferens-Biochemical and morphological characterization. Acta Physiol. Scand. Suppl. 493: 1-28.

Fried, G. (1982) Neuropeptide storage in vesicles. In Neurotransmitter Vesicles, R. L. Klein, H. Lagercrantz, and H. Zimmerman, eds., pp. 361-374, Academic Press, Inc., London.

Fried, G., H. Lagercrantz, and T. Hökfelt (1978) Improved isolation of small noradrenergic vesicles from rat seminal ducts following castration. Neuroscience 3: 1271-1291.

Fried, G., J. M. Lundberg, T. Hökfelt, H. Lagercrantz, J. Fahrenkrug, G. Lundgren, B. Holmstedt, E. Brodin, S. Efendić, and L. Terenius (1981a) Do peptides coexist with classical transmitters in the same neuronal storage vesicles? In Chemical Neurolrurusmission: 75 Years, L. Stjärne, H. Lagercrantz, and A. Wennmalm, eds., pp. 105-111, Academic Press, London.

Fried, G., $\AA$. Thureson-Klein, and H. Lagercrantz (1981b) Noradrenaline content correlated to matrix density in small noradrenergic vesicles from rat seminal ducts. Neuroscience 6:787-800.

Fried, G., H. Lagercrantz, R. L. Klein, and $\AA$. Thureson-Klein (1984) Large and small noradrenergic vesicles-Origin, contents and functional significance. In Catecholamines, E. Usdin, ed., pp. 45-53, Alan R. Liss, Inc., New York.

Grillo, M., and S. L. Palay (1962) Granule-containing vesicles in the autonomic nervous system. In Electron Microscopy, S. S. Breese, Jr., ed., Vol. 2, p. U-1, Academic Press, Inc., New York.

Gu, J., T. E. Adrian, K. Tatemoto, J. M. Polak, J. M. Allen, and S. R. Bloom (1983) Neuropeptide tyrosine (NPY), a major cardiac neuropeptide. Lancet 1: 1008-1010.

Hjemdahl, P., M. Daleskog, and T. Kahan (1979) Determination of plasma catecholamines by high performance liquid chromatography with electrochemical detection: Comparison with a radioenzymatic method. Life Sci. 25: 131-138.

Hökfelt, T. (1966) The effect of reserpine on the intraneuronal vesicles of the rat vas deferens. Experientia 22: 56.

Hökfelt, T. (1968) In vitro studies on central and peripheral monoamine neurons at the ultrastructural level. Z. Zellforsch. Mikrosk. Anat. 91: 1-74.

Hökfelt, T. (1969) Distribution of noradrenaline storing particles in peripheral adrenergic neurons as revealed by electron microscopy. Acta Physiol. Scand. 76: 427-440.

Hökfelt, T. (1973) Neuronal catecholamine storage vesicles. In Frontiers in Catecholamine Research, E. Usdin and S. Snyder, eds., pp. 439-446, Pergamon Press, New York. 
Hökfelt, T., O. Johansson, Å. Ljungdahl, J. M. Lundberg, and M. Schultzberg (1980) Peptidergic neurones. Nature 284: 515-521.

Klein, R. L., S. P. Wilson, D. J. Dzielak, W. -H. Yang, and O. H. Viveros (1982) Opioid peptides and noradrenaline co-exist in large dense-cored vesicles from sympathetic nerves. Neuroscience 7: 22552261.

Lundberg, J. M., and T. Hökfelt (1983) Coexistence of peptides and classical neurotransmitters. Trends Neurosci. 6: 325-333.

Lundberg, J. M., and K. Tatemoto (1982) Pancreatic polypeptide family (APP, BPP, NPY and PYY) in relation to sympathetic vasoconstriction resistant to $\alpha$-adrenoceptor blockade. Acta Physiol Scand. 116. 393-402.

Lundberg, J. M., G. Fried, J. Fahrenkrug, B. Holmstedt, T. Hökfelt, H. Lagercrantz, G. Lundgren, and A. Änggård (1981) Subcellular fractionation of cat submandibular gland: Comparative studies on the distribution of acetylcholine and vasoactive intestinal polypeptide (VIP). Neuroscience 6: 1001-1010.

Lundberg, J. M., T. Hökfelt, A. Änggård, L. Terenius, R. Elde, K Markey, M. Goldstein, and J. Kimmel (1982a) Organizational principles in the peripheral sympathetic nervous system: Subdivision by coexisting peptides (somatostatin-, avian pancreatic polypeptideand vasoactive intestinal polypeptide-like immunoreactive materials). Proc. Natl. Acad. Sci. U. S. A. 79: 1303-1307.

Lundberg, J. M., I. Terenius, T. Hökfelt, C. R. Martling, K. Tatemoto V. Mutt, J. Polak, S. Bloom, and M. Goldstein (1982b) Neuropeptide Y (NPY)-like immunoreactivity in peripheral noradrenergic neurons and effects of NPY on sympathetic function. Acta Physiol. Scand. 116: $477-480$

Lundberg, J. M., L. Terenius, T. Hökfelt, and M. Goldstein (1983) High levels of neuropeptide Y (NPY) in peripheral noradrenergic neurons in various mammals including man. Neurosci. Lett. 42: 167172.

Lundberg, J. M., L. Terenius, T. Hökfelt, and K. Tatemoto (1984) Comparative immunohistochemical and biochemical analysis of pancreatic polypeptide-like peptides with special reference to presence of neuropeptide $\mathrm{Y}$ in central and peripheral neurons. J. Neurosci. 4: 2376-2386.

Markey, K. A., S. Kondo, I. Shenkman, and M. Goldstein (1980) Purification and characterization of tyrosine hydroxcylase from a clonal phaeochromocytoma cell line. Mol. Pharmacol. 17: 79-85.

Norberg, K. A., P. L. Risley, and U. Ungerstedt (1966) Adrenergic innervation of the male reproductive ducts in some mammals. I. The distribution of adrenergic nerves. Z. Zellforsch. Mikrosk. Anat. 74: 182-197.

Otsuka, M., and I. 'Takahashi (1977) Putative peptide neurotransmitters. Annu. Rev. Pharmacol. Toxicol. 17: 425-439.

Pease, P. C. (1962) Buffered formaldehyde as a killing agent and primary fixative for electron microscopy. Anat. Res. 142: 342.

Pellegrino de Iraldi, A., and E. DeRobertis (1961) Action of reserpine on the submicroscopic morphology of the pineal gland. Experientia 17: $122-123$

Pfenninger, K. H. (1973) Synaptic morphology and cytochemistry. Prog. Histochem. Cytochem. 5: 1-86.

Pollard, R. M., M. Fillenz, and P. Kelly (1982) Parallel changes in ultrastructure and noradrenaline content of nerve terminals in rat vas deferens following transmitter release. Neuroscience 7: 16231629 .

Probert, L., J. De Mey, and J. M. Polak (1981) Distinct subpopulations of enteric $p$-type neurones contain substance $P$ and vasoactive intestinal polypeptide. Nature 294: 470-471.
Reynolds, S. E. (1963) The use of lead citrate at high $\mathrm{pH}$ as an electronopaque stain in electron microscopy. J. Cell Biol. 17: 208-212.

Richardson, K. C. (1962) The fine structure of autonomic nerve endings of rat vas deferens. J. Anat. 96: 427-442.

Richardson, K. C. (1964) The fine structure of the albino rabbit iris with special reference of the adrenergic and cholinergic nerves and nerve endings in its intrinsic muscles. Am. J. Anat. 111: 173-205.

Richardson, K. C. (1966) Electron microscopic identification of autonomic nerve endings. Nature New Biol. 210: 756.

Sjöstrand, N. O. (1965) The adrenergic innervation of the vas deferens and the accessory male genital glands. Acta Physiol. Scand. 65 (Suppl. 257): 1-82.

Snyder, S. H. (1980) Brain peptides as neurotransmitters. Science 209: 976-983.

Sundler, F., E. Moghimzadeh, R. Håkanson, M. Ekelund, and P. Emson (1983) Nerve fibers in the gut and pancreas of the rat displaying neuropeptide-Y immunoreactivity. Intrinsic and extrinsic origin. Cell Tissue Res. 230: 487-493.

Tapia, F. J., I. M. Varndell, L. Probert, J. De Mey, and J. M. Polak (1983) Double immunogold staining method for the simultaneous ultrastructural localization of regulatory peptides. J. Histochem. Cytochem. 31: 977-981.

Tatemoto, K. (1982) Neuropeptide Y: Complete amino acid sequence of the brain peptide. Proc. Natl. Acad. Sci. U. S. A. 79: 5485-5489.

Tatemoto, K., M. Carlquist, and V. Mutt (1982) Neuropeptide Y-A novel brain peptide with structural similarities to peptide YY and pancreatic polypeptide. Nature 296: 659-660.

Taxi, J. (1965) Contribution a l'étude des connexions des neurones moteurs du système nerveux autonomie. Ann. Sci. Nat. Zool. Biol. Anim. 12: 413-674.

Terenghi, G., J. M. Polak, J. M. Allen, S. Q. Zhang, W. G. Unger, and S. R. Bloom (1983) Neuropeptide Y-immunoreactive nerves in the uvea of guinea pig and rat. Neurosci. Lett. 42: 33-38.

Thureson-Klein, A. (1983) Exocytosis from large and small dense cored vesicles in noradrenergic nerve terminals. Neuroscience 10: 245-252.

Thureson-Klein, Å., S. H. Chen-Yen, and R. L. Klein (1974) Retention of matrix density in adrenergic vesicles after extensive norepinephrine depletion. Experientia 30: 935-937.

'Tranzer, J. P., and J. G. Richards (1976) Ultrastructural cytochemistry of biogenic amines in nervous tissue: Methodological improvements. J. Histochem. Cytochem. 24: 1178-1193.

Tranzer, J. G., and H. Thoenen (1967) Electron microscopic localization of 5-hydroxydopamine (3,4,5-trihydroxy-phenylethylamine), a new "false" sympathetic transmitter. Experientia 23:123-124.

Tranzer, J. P., and H. Thoenen (1968) Various types of amine-storing vesicles in peripheral adrenergic nerve terminals. Experientia 24: 484-486.

Van Orden, L. S., III, F. E. Bloom, J. R. Barrnett, and N. J. Giarman (1966) Histochemical and functional relationships of catecholamines in adrenergic nerve endings. I. Participation of granular vesicles. J. Pharmacol. Exp. Ther. 154: 185-199.

Van Orden, L. S., III, K. G. Bensch, and N. J. Giarman (1967) Histochemical and functional relationships of catecholamines in adrenergic nerve endings.II. Extravesicular norepinephrine. J. Pharmacol. Exp. Ther. 155: 428-439.

von Euler, U. S., and N. A. Hillarp (1956) Evidence for the presence of noradrenaline in submicroscopic structures of adrenergic axons. $\mathrm{Na}$ ture New Biol. 177: 44-45.

Wilson, S. P., R. L. Klein, K. -J. Chang, M. S. Gasparis, O. H. Viveros, and W. $-\mathrm{H}$. Yang (1980) Are opioid peptides co-transmitters in noradrenergic vesicles of sympathetic nerves? Nature 288: 707-709. 